\title{
Critical role of the cholinergic system for object-in-place associative recognition memory
}

\author{
Gareth R.I. Barker and Elizabeth C. Warburton ${ }^{1}$ \\ MRC Centre for Synaptic Plasticity, Department of Anatomy, University of Bristol, Bristol BS8 1TD, United Kingdom
}

\begin{abstract}
Object-in-place memory, which relies on the formation of associations between an object and the place in which it was encountered, depends upon a neural circuit comprising the perirhinal (PRH) and medial prefrontal (mPFC) cortices. This study examined the contribution of muscarinic cholinergic neurotransmission within this circuit to such object-in-place associative memory. Intracerebral administration of scopolamine in the PRH or mPFC impaired memory acquisition, but not retrieval and importantly we showed that unilateral blockade of muscarinic receptors simultaneously in both regions in opposite hemispheres, significantly impaired performance. Thus, object-in-place associative memory depends upon cholinergic modulation of neurones within the PRH-PFC circuit.
\end{abstract}

Recognition memory enables individuals to judge whether stimuli have been encountered before. In its most basic form such judgments may be made on the basis of simply whether a stimulus is familiar or novel (familiarity discrimination). However, these judgments may also be made using associations formed between a stimulus and the location or environmental setting in which it was previously encountered. Such object-in-place associative memory in animals is of particular interest as it is acquired rapidly and it requires the integration of object and spatial information and thus has been described as an analog of human episodic memory (Wilson et al. 2008).

The perirhinal cortex (PRH) in the medial temporal lobe is a critical neural structure for object recognition and object-inplace associative memory (Bussey et al. 2000; Barker et al. 2007), but unlike object recognition, this memory process is also dependent on the medial prefrontal cortex (mPFC) (Kesner and Ragozzino 2003; Browning et al. 2005; Barker et al. 2007) and crucially it has been shown to depend upon a functional interaction between the PRH and $\mathrm{mPFC}$, with each region making a distinct cognitive contribution to the memory formation (Barker et al. 2007; Barker and Warburton 2008).

Having identified two neural regions critical for object-inplace associative memory, we now extend our investigations to explore the underlying cellular mechanisms mediating acquisition or retrieval of this memory process. The present study focused on the neurotransmitter acetylcholine as cholinergic innervation of the PRH is crucial for familiarity discrimination (Tang et al. 1997; Easton and Gaffan 2001; Warburton et al. 2003; Abe et al. 2004; Winters and Bussey 2005). In contrast, the role of muscarinic receptor neurotransmission in the $\mathrm{PRH}$ or $\mathrm{mPFC}$ in object-inplace associative memory is unknown. Further, while it might appear that object recognition memory and object-in-place memory are likely to share common neural substrates, recent data from our laboratory suggest that this may not be the case (Griffiths et al. 2008).

To explore the importance of muscarinic cholinergic neurotransmission within the PRH-mPFC circuit for object-in-place memory, rats were implanted with bilateral cannulae aimed at the PRH or mPFC or both regions to allow direct intracerebral administration of scopolamine during distinct stages of an objectin-place task. Memory performance was tested following either a short $(5 \mathrm{~min})$ or long $(1 \mathrm{~h})$ retention delay. All animal procedures

\footnotetext{
'Corresponding author.
}

E-mail e.c.warburton@bristol.ac.uk; fax (44) 117-9291687. Article is online at http://www.learnmem.org/cgi/doi/10.1101/lm.1121309. were performed in accordance with the United Kingdom Animals Scientific Procedures Act (1986) and associated guidelines. Details of the surgery, infusion procedures, behavioral testing, and histology have been published previously (Barker and Warburton 2008). Briefly, male DA rats (230-250 g, Bantin and Kingman, UK) housed under a 12-h/12-h light/dark cycle (light phase 18:00-6:00 h), were anesthetized with isoflurane (induction $4 \%$, maintenance $2 \%-3 \%$ ) and surgically implanted with bilateral cannulae aimed at either the PRH or mPFC or both regions. After a two-week recovery period all rats were handled, habituated, and then tested in the object-in-place memory task.

Sample phase: Each rat was placed in a black open-topped wooden arena $(50 \times 90 \times 100 \mathrm{~cm})$ containing four different objects (A, B, C, D) constructed from "Duplo" (Lego UK Ltd.). The walls of the arena were surrounded with a black cloth to a height of $1.5 \mathrm{~m}$, and the floor covered with sawdust. The objects were placed $15 \mathrm{~cm}$ from the walls (see Fig. 1A) and each rat was allowed to explore the objects for $5 \mathrm{~min}$, after which it was removed for the delay ( $5 \mathrm{~min}$ or $1 \mathrm{~h}$ ). Exploratory behavior was defined as the animal directing its nose toward the object at a distance of $<2 \mathrm{~cm}$. Any other behavior, such as looking around while sitting on or resting against the object, was not recorded. Subjects that failed to complete a minimum of 15-s exploration in the sample phase or $10 \mathrm{~s}$ of exploration in the test phase were excluded from the analysis.

Test phase: Two of the objects, e.g., B and D, exchanged positions and the subjects were replaced in the arena for $3 \mathrm{~min}$. The time spent exploring the two objects that had changed position was compared to the time spent exploring the two objects that had remained in the same position. If object-in-place memory is intact, subjects spend more time exploring the "moved" objects, compared to the "unmoved" objects. Scopolamine hydrobromide (Sigma-Aldrich) dissolved in sterile $0.9 \%$ saline solution was administered at a dose of $10 \mu \mathrm{g} / \mu \mathrm{L}$ per hemisphere (Schroeder and Packard 2002; Warburton et al. 2003; Winters et al. 2006); control infusions consisted of saline. The infusions were given either $15 \mathrm{~min}$ before the sample phase or $15 \mathrm{~min}$ before the test phase. At the end of the experiment, each rat was anesthetized and perfused transcardially. Coronal brain sections $(40 \mu \mathrm{m})$ were stained with cresyl-violet to verify the cannulae locations. All the rats in the PRH group had the tip of the bilateral cannulae in the $\mathrm{PRH}$ and all the rats in the mPFC group had tips in the ventral portion of the prelimbic or dorsal portion of the infralimbic region of the prefrontal cortex (Fig. 1B). From unpublished observations, using Indian ink and radiolabeled scopolamine, the region infused is estimated to be $1-1.5 \mathrm{~mm}^{3}$, and largely confined to perirhinal 


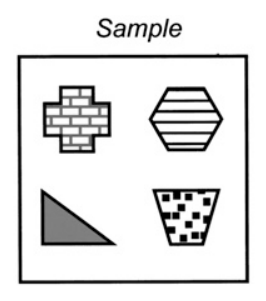

A

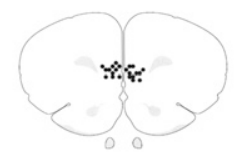

B
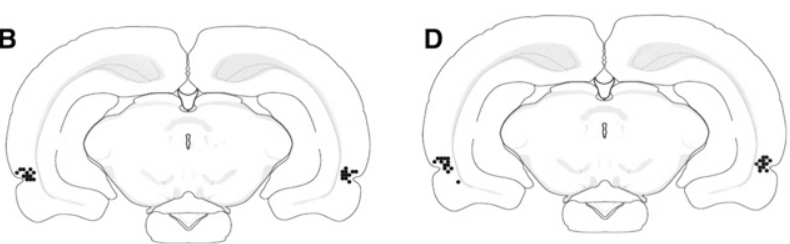

Figure 1. Diagrammatic representations of the individual infusion sites in each animal. $(A)$ Bilateral medial prefrontal (mPFC) group. (B) Bilateral perirhinal (PRH) group. (C) The mPFC infusion sites of the $\mathrm{PRH}+\mathrm{mPFC}$ group. (D) The PRH infusion sites of the PRH+mPFC group. All of the infusion sites were within the PRH or $\mathrm{MPFC}$.

cortex or the prelimbic/infralimbic regions of the prefrontal cortex. This spread is consistent with previously quoted results in other brain regions (Martin 1991; Izquierdo et al. 2000; Attwell et al. 2001).

Figure 2, A and B show the performance of the rats receiving bilateral infusions of scopolamine or vehicle into either the PRH $(n=12)$ or mPFC $(n=12) 15$ min prior to the sample phase. After a minimum of $48 \mathrm{~h}$, vehicle or scopolamine was infused in a crossover design and the animal retested using different objects. A three-way ANOVA (drug $\times$ region $\times$ delay) showed that scopolamine infusion into either region significantly impaired the acquisition of object-in-place memory (main effect of drug $F_{(1,35)}=$ 63.87, $P<0.001$ ). The magnitude of the deficit was similar irrespective of the region into which scopolamine was infused (region $F_{(1,35)}<1.0$ ) or the delay employed (delay $F_{(1,35)}<1.0$ ). Further analyses to examine whether individual groups discriminated between the objects, using a within subjects $t$-test (twotailed), confirmed that vehicle-treated animals in the PRH and mPFC groups showed a significant preference for the moved objects over the objects that had remained in the same position, irrespective of the retention delay (PRH $5 \min t_{(9)}=2.96, P<0.02$; $1 \mathrm{~h} t_{(10)}=5.71, P<0.001: \operatorname{mPFC} 5 \min t_{(5)}=5.47, P<0.005 ; 1 \mathrm{~h}$ $t_{(11)}=9.89, P<0.001$ ), while scopolamine infusion into the PRH or mPFC significantly disrupted the animal's ability to discriminate $\left(\right.$ PRH $5 \min t_{(9)}=0.13, P=0.9 ; 1 \mathrm{~h} t_{(10)}=0.92, P=0.38: \mathrm{mPFC} 5 \mathrm{~min}$ $\left.t_{(5)}=0.051, P=0.961 ; 1 \mathrm{~h} t_{(11)}=0.68, P=0.51\right)$. Scopolamine was without effect on the total amount of exploration completed in the sample or test phases (all Fs $<1.0$ ).

It could be argued that the impairment produced by intracortical infusions of scopolamine following a short delay, reflects an effect on retrieval as well as acquisition. Therefore, we examined the effect of pretest administration of scopolamine (infusion 15 min before the start of the test phase) in the mPFC or PRH following a $1 \mathrm{~h}$ delay. No significant impairments were found (mean discrimination ratio \pm SEM: PRH vehicle $0.38 \pm 0.07$, scopolamine $0.46 \pm 0.11$; mPFC vehicle $0.37 \pm 0.08$, scopolamine $0.44 \pm 0.05$ ), confirmed by a nonsignificant drug effect $\left(F_{(1,14)}<1.0, P>0.1\right)$ and nonsignificant drug $\times$ area interaction
$\left(F_{(1,14)}=<1.0, P>0.1\right)$. In addition all groups significantly discriminated between the moved objects compared to objects in the same location (PRH vehicle $t_{(7)}=4.95, P<0.01$; $\mathrm{PRH}$ scopolamine $t_{(7)}=3.45, P<0.05 ; \mathrm{mPFC}$ vehicle $t_{(7)}=4.26, P<0.01$; mPFC scopolamine $\left.t_{(7)}=8.37, P<0.01\right)$. Scopolamine was without effect on the total amount of exploration completed in the test phase (drug $\times$ region $\left.F_{(1,14)}<1.0, P>0.05\right)$.

To evaluate the importance of intrahemispheric interactions between these cortical regions and the cholinergic system, a third group of animals had cannulae implanted into both the PRH and mPFC $(n=12)$. In this experiment the behavioral effects of unilateral scopolamine infusions into the PRH and mPFC in the same hemisphere (Scop Ipsi) were compared with the effects of unilateral scopolamine infusions into opposite hemispheres (Scop Contra). The animals assigned to the Scop Ipsi group on day one, received infusions into opposite hemispheres (Scop Contra) on day two (minimum of $48 \mathrm{~h}$ later). Likewise, the animals in the Scop Contra group on day one, received ipsilateral infusions on day two. Figure 3 shows discrimination performance following a 5 min or $1 \mathrm{~h}$ delay. A two-way within-subject ANOVA revealed that the Scop Contra group was significantly impaired (infusion $F_{(1,20)}=44.35, P<0.001$ ) irrespective of the delay (infusion $\times$ delay $\left.F_{(1,20)}<1.0, P<0.05\right)$. Further analysis confirmed that

A

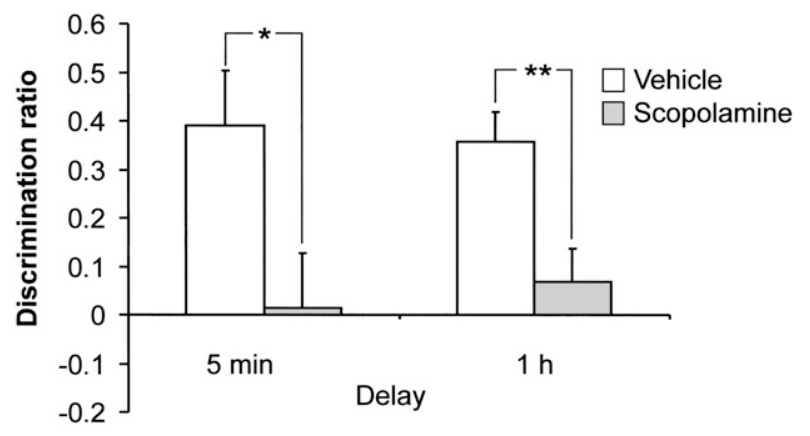

B

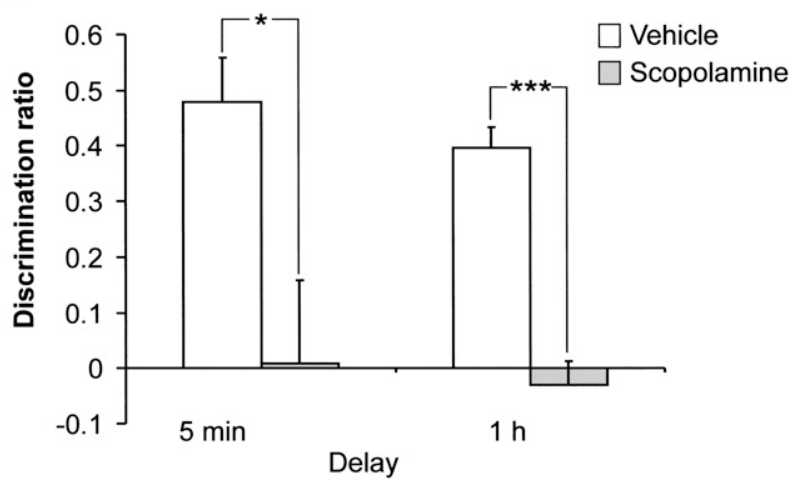

Figure 2. Discrimination between the objects was calculated using a discrimination ratio, which takes into account individual differences in the total amount of exploration. The discrimination ratio is calculated as follows: the difference in time spent by each animal exploring objects that changed position compared to the objects that remained in the same position divided by the total time spent exploring all objects. (A) Infusion of scopolamine (Scop) into the perirhinal cortex (PRH) significantly impaired performance in the object-in-place task following a $5 \mathrm{~min}$ and a $1 \mathrm{~h}$ delay. (B) Infusion of scopolamine (Scop) into the medial prefrontal cortex (mPFC) significantly impaired performance in the objectin-place task following a $5 \mathrm{~min}$ and a $1 \mathrm{~h}$ delay. Illustrated for each group is the mean (+ SEM) discrimination ratio. ${ }^{*} P<0.05$; ${ }^{* *} P<0.01$; and ${ }^{* * *} P<0.001$ difference between groups. 


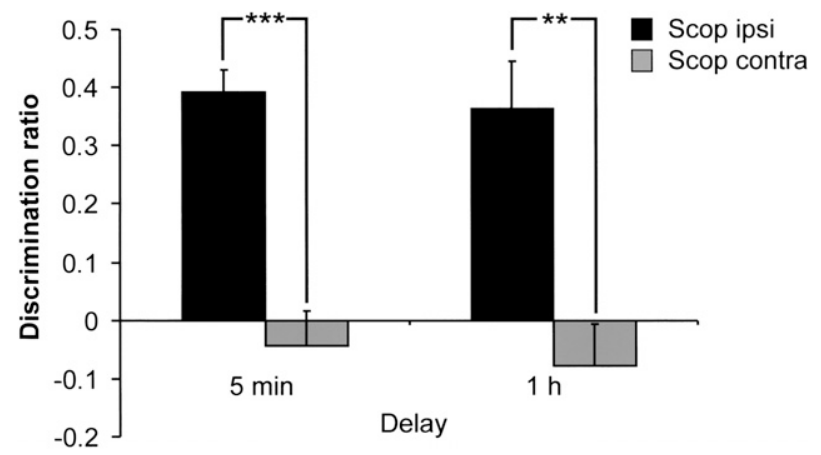

Figure 3. Unilateral scopolamine infusions into the PRH and mPFC in opposite hemispheres (Scop Contra) impaired object-in-place performance following both a 5 min and a $1 \mathrm{~h}$ delay. Scopolamine infusions into both the PRH and mPFC in the same hemisphere (Scop Ipsi) had no effect on performance following either delay. ${ }^{* *} P<0.01$ and ${ }^{* * *} P<0.001$ difference between groups.

the Scop Contra group failed to discriminate between the moved and unmoved objects $\left(5 \min t_{(10)}=0.70, P>0.1 ; 1 \mathrm{~h}\right.$ $t_{(10)}=1.03, P>0.1$, while the Scop Ipsi group preferentially explored the moved objects $\left(5 \min t_{(10)}=9.99, P<0.0001 ; 1 \mathrm{~h}\right.$ $\left.t_{(10)}=4.34, P=0.001\right)$.

Scopolamine was without effect on overall exploration levels during the sample (infusion $\times$ delay $F_{(1,20)}<1.0, P>0.05$; infusion $F_{(1,20)}<1.0, P>0.05$; delay $\left.F_{(1,20)}<1.0, P>0.05\right)$ or test phases (infusion $\times$ delay $F_{(1,20)}<1.0, P>0.05$; infusion $F_{(1,20)}<$ $1.0, P>0.05)$. There was a significant main effect of delay $\left(F_{(1,20)}=\right.$ 10.67, $P<0.01)$, as the Scop Ipsi and Scop Contra groups completed a greater amount of exploration in the test phase following a $1 \mathrm{~h}$ delay compared to a 5 min delay.

These results demonstrate that acquisition, but not retrieval of object-in-place memory, is dependent upon muscarinic cholinergic neurotransmission in both the MPFC and PRH. Thus, acute bilateral administration of scopolamine directly into the mPFC or PRH before the sample phase impaired both short- and long-term memory performances. In contrast administration of scopolamine into either the mPFC or PRH prior to the test phase had no effect. Significantly, co-administration of scopolamine into the PRH and mPFC in opposite hemispheres produced a significant impairment in both short-term and long-term object-in-place memory compared to performance following co-administration of scopolamine into the PRH and mPFC in the same hemisphere. Thus, concomitant activation of cholinergic muscarinic receptors is necessary in both regions for the formation of object-in-place associative recognition memory.

Our previous studies investigating the role of the mPFC and $\mathrm{PRH}$ in object-in-place associative memory suggest that these regions make different cognitive contributions to this mnemonic process. Thus, the PRH appears to be primarily involved in the acquisition of "object" information, while we have hypothesized that the role of the mPFC is to integrate object and place information (Barker et al. 2007). As administration of scopolamine into either region disrupted performance following a long- or short-retention delay, the present data suggest that the neural mechanisms underlying both these different cognitive processes must be dependent upon cholinergic neurotransmission.

The results demonstrate that muscarinic receptor neurotransmission is clearly critical for acquisition of the object-in-place task as no impairment was produced when scopolamine was administered only prior to the test phase. While the current study is the first to investigate the importance of cholinergic neurotransmission in object-in-place associative memory, a number of previous studies have shown that intra-PRH infusions of scopolamine block discrimination of novel and familiar objects when administered prior to the sample phase, but not when administered immediately after the sample phase or prior to the test phase (Aigner and Mishkin 1986; Aigner et al. 1991; Warburton et al. 2003; Winters et al. 2006). Thus, together these results support the hypothesis that muscarinic cholinergic neurotransmission within the PRH is necessary for encoding representations of new visual stimuli for subsequent recognition (Turchi et al. 2005), but not for the retrieval of such information. The present results also show for the first time that muscarinic receptor neurotransmission within the mPFC is crucial for the encoding, but not the retrieval of object-in-place memory.

It may be argued that the disruptions in performance following administration of scopolamine reflect disruptions in attentional processing. Indeed muscarinic cholinergic neurotransmission in the prefrontal cortex has been implicated in both mnemonic and attentional processes (Voytko et al. 1994; Everitt and Robbins 1997; Chudasama et al. 2004; Dalley et al. 2004). However, deficits in attentional processing are typically observed when the attentional demands of the tasks are high, for example, when very short (millisecond) stimulus exposure times are used (Chudasama et al. 2004; Dalley et al. 2004). In the present study, the exposure time to the stimuli is relatively long (minutes); further there was no evidence of a drug-associated change in explorative behavior following either an infusion into the mPFC or PRH or simultaneously into both regions. Thus, it seems unlikely that the impairments in memory observed can be attributed purely to an attentional deficit, although it is possible that during the encoding of the object-in-place task attentional processes are also recruited involving the cholinergic afferents to the mPFC or PRH.

The results showing that simultaneous muscarinic cholinergic blockade in the PRH and mPFC produces a significant impairment in performance support our previous findings of a neural system for object-in-place memory and extend these findings to show that cholinergic neurotransmission is a key component within the system. Our results also support those studies in primates demonstrating a circuit involving the basal forebrain, frontal cortex, and inferior temporal cortex is necessary for object memory encoding (Easton et al. 2002; Easton and Parker 2003).

Results from our laboratory have shown that the maintenance of long-term, but not short-term, object-in-place memory is critically dependent upon concurrent NMDA receptor activation in the PRH and mPFC (Barker and Warburton 2008), while shortterm object-in-place performance is dependent upon kainate receptor activation in the PRH. Hence, we have argued that there may be multiple cellular mechanisms underlying encoding of information for the short or long term. The present study contrasts with these findings as it demonstrates the necessity for muscarinic receptor activation for both short- and long-term object-in-place memory. Primate studies have indicated that a synergistic interaction between the cholinergic and glutamatergic systems plays an important role in the regulation of visual recognition memory (Matsuoka and Aigner 1996). Hence, further investigations are warranted to explore such interactions in the rat; for example, an interaction between NMDA and muscarinic receptor neurotransmission may mediate long-term recognition memory, while a kainate-muscarinic receptor interaction may mediate short-term recognition memory. Further, the extent to which the contribution of the cholinergic system to encoding of object-in-place memory within the PRH-mPFC system is the same for both shortor long-term memory is unknown.

Our results have demonstrated that when a subject is required to use information concerning an association between an object and a place to produce a behavioral response, muscarinic 
cholinergic receptors in the mPFC are involved. Further, the object-in-place task requires the subject to acquire and remember the topographical relationship between the objects, a process that is known to depend upon the parietal cortex (Goodrich-Hunsaker et al. 2005). The precise contribution of object and spatial information processing in the parietal cortex to the operation of the PRH-mPFC circuit has yet to be determined.

In conclusion, the cholinergic projections to the PRH and mPFC originating in the basal forebrain (Wenk et al. 1980) are an important component of the neural mechanisms underlying short- and long-term object-in-place associative memory.

\section{Acknowledgments}

This work was supported by the Biotechnology and Biological Sciences Research Council. We thank Jane Robbins and Katherine Narduzzo for assistance with histology. We also thank Professor Malcolm Brown for helpful comments on an earlier draft of this manuscript.

\section{References}

Abe, H., Ishida, Y., and Iwasaki, T. 2004: Perirhinal N-methyl-D-aspartate and muscarinic systems participate in object recognition in rats. Neurosci. Lett. 356: 191-194.

Aigner, T.G. and Mishkin, M. 1986: The effects of physostigmine and scopolamine on recognition memory in monkeys. Behav. Neural Biol. 45: $81-87$.

Aigner, T.G., Mitchell, S.J., Aggleton, J.P., DeLong, M.R., Struble, R.G., Price, D.L., Wenk, G.L., Pettigrew, K.D., and Mishkin, M. 1991: Transient impairment of recognition memory following ibotenic-acid lesions of the basal forebrain in macaques. Exp. Brain Res. 86: 18-26.

Attwell, P.J., Rahman, S., and Yeo, C.H. 2001: Acquisition of eyeblink conditioning is critically dependent on normal function in cerebellar cortical lobule HVI. J. Neurosci. 21: 5715-5722.

Barker, G.R.I. and Warburton, E.C. 2008: NMDA receptor plasticity in the perirhinal and prefrontal cortices is crucial for the acquisition of longterm object-in-place associative memory. J. Neurosci. 28: 2837-2844.

Barker, G.R.I., Bird, F., Alexander, V., and Warburton, E.C. 2007: Recognition memory for objects, place, and temporal order: A disconnection analysis of the role of the medial prefrontal cortex and perirhinal cortex. J. Neurosci. 27: 2948-2957.

Browning, P.G., Easton, A., Buckley, M.J., and Gaffan, D. 2005: The role of prefrontal cortex in object-in-place learning in monkeys. Eur. J. Neurosci. 22: 3281-3291.

Bussey, T.J., Duck, J., Muir, J.L., and Aggleton, J.P. 2000: Distinct patterns of behavioural impairments resulting from fornix transection or neurotoxic lesions of the perirhinal and postrhinal cortices in the rat. Behav. Brain Res. 111: 187-202.

Chudasama, Y., Dalley, J.W., Nathwani, F., Bouger, P., and Robbins, T.W. 2004: Cholinergic modulation of visual attention and working memory: Dissociable effects of basal forebrain 192-IgG-saporin lesions and intraprefrontal infusions of scopolamine. Learn. Mem. 11: 78-86.

Dalley, J.W., Theobald, D.E., Bouger, P., Chudasama, Y., Cardinal, R.N., and Robbins, T.W. 2004: Cortical cholinergic function and deficits in visual attentional performance in rats following 192 IgG-saporin-induced lesions of the medial prefrontal cortex. Cereb. Cortex 14: 922-932.

Easton, A. and Gaffan, D. 2001: Crossed unilateral lesions of the medial forebrain bundle and either inferior temporal or frontal cortex impair object-reward association learning in Rhesus monkeys. Neuropsychologia 39: 71-82.

Easton, A. and Parker, A. 2003: A cholinergic explanation of dense amnesia. Cortex 39: 813-826.

Easton, A., Ridley, R.M., Baker, H.F., and Gaffan, D. 2002: Unilateral lesions of the cholinergic basal forebrain and fornix in one hemisphere and inferior temporal cortex in the opposite hemisphere produce severe learning impairments in rhesus monkeys. Cereb. Cortex 12: 729-736.

Everitt, B.J. and Robbins, T.W. 1997: Central cholinergic systems and cognition. Annu. Rev. Psychol. 48: 649-684.

Goodrich-Hunsaker, N.J., Hunsaker, M.R., and Kesner, R.P. 2005: Dissociating the role of the parietal cortex and dorsal hippocampus for spatial information processing. Behav. Neurosci. 119: 1307-1315.

Griffiths, S., Scott, H., Glover, C., Bienemann, A., Ghorbel, M.T., Uney, J., Brown, M.W., Warburton, E.C., and Bashir, Z.I. 2008: Expression of long-term depression underlies visual recognition memory. Neuron 58: 186-194.

Izquierdo, L.A., Barros, D.M., Ardenghi, P.G., Pereira, P., Rodrigues, C., Choi, H., Medina, J.H., and Izquierdo, I. 2000: Different hippocampal molecular requirements for short- and long-term retrieval of one-trial avoidance learning. Behav. Brain Res. 111: 93-98.

Kesner, R.P. and Ragozzino, M.E. 2003: The role of the prefrontal cortex in object-place learning: A test of the attribute specificity model. Behav. Brain Res. 146: 159-165.

Martin, J.H. 1991: Autoradiographic estimation of the extent of reversible inactivation produced by microinjection of lidocaine and muscimol in the rat. Neurosci. Lett. 127: 160-164.

Matsuoka, N. and Aigner, T.G. 1996: Cholinergic-glutamatergic interactions in visual recognition memory of rhesus monkeys. Neuroreport 7: 565-568.

Schroeder, J.P. and Packard, M.G. 2002: Post-training intra-basolateral amygdala scopolamine impairs food- and amphetamine-induced conditioned place preferences. Behav. Neurosci. 116: 922-927.

Tang, Y., Mishkin, M., and Aigner, T.G. 1997: Effects of muscarinic blockade in perirhinal cortex during visual recognition. Proc. Natl. Acad. Sci. 94: 12667-12669.

Turchi, J., Saunders, R.C., and Mishkin, M. 2005: Effects of cholinergic deafferentation of the rhinal cortex on visual recognition memory in monkeys. Proc. Natl. Acad. Sci. 102: 2158-2161.

Voytko, M.L., Olton, D.S., Richardson, R.T., Gorman, L.K., Tobin, J.R., and Price, D.L. 1994: Basal forebrain lesions in monkeys disrupt attention but not learning and memory. J. Neurosci. 14: 167-186.

Warburton, E.C., Koder, T., Cho, K., Massey, P.V., Duguid, G., Barker, G.R.I., Aggleton, J.P., Bashir, Z.I., and Brown, M.W. 2003: Cholinergic neurotransmission is essential for perirhinal cortical plasticity and recognition memory. Neuron 38: 987-996.

Wenk, H., Bigl, V., and Meyer, U. 1980: Cholinergic projections from magnocellular nuclei of the basal forebrain to cortical areas in rats. Brain Res. Rev. 2: 295-316.

Wilson, C.R., Baxter, M.G., Easton, A., and Gaffan, D. 2008: Addition of fornix transection to frontal-temporal disconnection increases the impairment in object-in-place memory in macaque monkeys. Eur. J. Neurosci. 27: 1814-1822.

Winters, B.D. and Bussey, T.J. 2005: Removal of cholinergic input to perirhinal cortex disrupts object recognition but not spatial working memory in the rat. Eur. J. Neurosci. 21: 2263-2270.

Winters, B.D., Saksida, L.M., and Bussey, T.J. 2006: Paradoxical facilitation of object recognition memory after infusion of scopolamine into perirhinal cortex: Implications for cholinergic system function. $J$. Neurosci. 26: 9520-9529.

Received June 25, 2008; accepted in revised form October 16, 2008. 


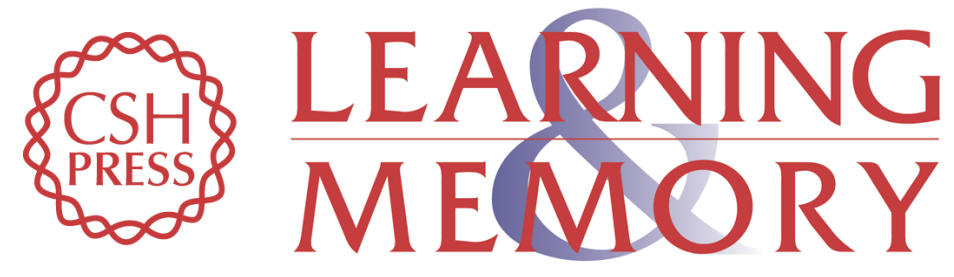

\section{Critical role of the cholinergic system for object-in-place associative recognition memory}

Gareth R.I. Barker and Elizabeth C. Warburton

Learn. Mem. 2009, 16:

Access the most recent version at doi:10.1101//m.1121309

References This article cites 29 articles, 8 of which can be accessed free at: http://learnmem.cshlp.org/content/16/1/8.full.html\#ref-list-1

License

Email Alerting Receive free email alerts when new articles cite this article - sign up in the box at the Service top right corner of the article or click here. 